\title{
Cardiac Troponin Mutations and Restrictive Cardiomyopathy
}

\author{
Michelle S. Parvatiyar, Jose Renato Pinto, David Dweck, and James D. Potter \\ Department of Molecular and Cellular Pharmacology, Miller School of Medicine, University of Miami, Room 6085A, RMSB, \\ 1600 NW 10th Avenue, Miami, FL 33136, USA
}

Correspondence should be addressed to James D. Potter, jdpotter@miami.edu

Received 13 November 2009; Accepted 22 February 2010

Academic Editor: Aikaterini Kontrogianni-Konstantopoulos

Copyright ( $) 2010$ Michelle S. Parvatiyar et al. This is an open access article distributed under the Creative Commons Attribution License, which permits unrestricted use, distribution, and reproduction in any medium, provided the original work is properly cited.

\begin{abstract}
Mutations in sarcomeric proteins have recently been established as heritable causes of Restrictive Cardiomyopathy (RCM). RCM is clinically characterized as a defect in cardiac diastolic function, such as, impaired ventricular relaxation, reduced diastolic volume and increased end-diastolic pressure. To date, mutations have been identified in the cardiac genes for desmin, $\alpha$-actin, troponin I and troponin T. Functional studies in skinned muscle fibers reconstituted with troponin mutants have established phenotypes consistent with the clinical findings which include an increase in myofilament $\mathrm{Ca}^{2+}$ sensitivity and basal force. Moreover, when RCM mutants are incorporated into reconstituted myofilaments, the ability to inhibit the ATPase activity is reduced. A majority of the mutations cluster in specific regions of cardiac troponin and appear to be mutational "hot spots". This paper highlights the functional and clinical characteristics of RCM linked mutations within the troponin complex.
\end{abstract}

\section{Introduction}

Cardiac contractility is governed by the thin filament regulatory proteins, cardiac troponin (cTn) and tropomyosin $(\mathrm{Tm})$, and available levels of intracellular free calcium $\left(\left[\mathrm{Ca}^{2+}\right]_{i}\right)$. Cardiac troponin is located at regular intervals along the thin filament and consists of three subunits: cardiac troponin $\mathrm{C}(\mathrm{cTnC})$, troponin $\mathrm{I}(\mathrm{cTnI})$, and troponin $\mathrm{T}$ (cTnT). The cTn complex plays an important role in the regulation of striated muscle contraction. Within the $\mathrm{cTn}$ complex, cTnC acts as a $\mathrm{Ca}^{2+}$ sensor which confers $\mathrm{Ca}^{2+}$ sensitivity to muscle contraction [1]. Cardiac TnI is the inhibitory subunit, primarily functioning to prevent actin and myosin from interacting in the absence of $\mathrm{Ca}^{2+}$. The cTnT subunit binds to tropomyosin $(\mathrm{Tm})$ and is responsible for transmitting the $\mathrm{Ca}^{2+}$ binding signal from cTn to $\mathrm{Tm}$. During systole, the rise in $\left[\mathrm{Ca}^{2+}\right]_{i}$ allows binding of $\mathrm{Ca}^{2+}$ to the regulatory site of $\mathrm{cTnC}$ which leads to the movement of $\mathrm{Tm}$ over the actin filament. This permits myosin to interact with actin, subsequently leading to the generation of muscle tension [2]. Alternatively in diastole, the $\left[\mathrm{Ca}^{2+}\right]_{i}$ levels decline and $\mathrm{Ca}^{2+}$ dissociates from cTnC, allowing $\mathrm{Mg}^{2+}$ to bind to the $\mathrm{C}$-terminal sites. This enables the muscle to relax and allows ventricular filling to occur. Currently, only cTnI and $\mathrm{CTnT}$ have been linked to restrictive cardiomyopathy (RCM) since no mutations have been identified in $\mathrm{cTnC}$ yet. Notably, mutations in sarcomeric proteins have the potential to disrupt essential thin filament interactions which can lead to the enhanced $\mathrm{Ca}^{2+}$ sensitivity of contraction, arrhythmogenesis, cardiomyopathy and even sudden cardiac death (SCD) [3]. This paper will address the clinical aspects, as well as the functional studies reported to date for the known troponin mutations associated with RCM.

\section{Troponin and Cardiac Muscle Regulation}

The functional roles of the individual cTn subunits are distinct and involve complex short and long range proteinprotein interactions. The binding of $\mathrm{Ca}^{2+}$ to the regulatory site of $\mathrm{cTnC}$ evokes the minimal exposure of a hydrophobic cleft within the $\mathrm{N}$-domain of cTnC. This enables the regulatory region of $\mathrm{c} \operatorname{TnI}$ (residues 148-163) to associate with $\mathrm{cTnC}[4,5]$. Structural analysis has determined that cTnI interacts with cTnC in an antiparallel manner [5, 6]. The association of cTnI with cTnC in the presence of $\mathrm{Ca}^{2+}$ facilitates the release of cTnI's inhibitory region (residues 128-147) from actin [7]. When cTnI dissociates from actin, Tm changes its conformation and allows for 
the full interaction of myosin heads with actin [1]. Current models propose that thin filament activation is regulated by $\mathrm{Ca}^{2+}$ binding to $\mathrm{cTnC}$ which influences the number of accessible cross-bridges by shifting the equilibrium from weakly (nonforce-producing) to strongly (force-producing) bound cross-bridges $[8,9]$.

Cardiac TnI has a unique feature absent in the fast and slow skeletal muscle isoforms such that it possesses a 30amino acid $\mathrm{N}$-terminal extension which contains two Protein Kinase A (PKA) phosphorylation sites (Ser 22 and 23) [10, 11]. Activation of PKA by $\beta$-adrenergic stimulation enhances the rate of cross-bridge cycling and increases the rate of $\mathrm{Ca}^{2+}$ uptake by the sarcoplasmic reticulum [12-14]. Both of these phenomena contribute to the lusitropic effect associated with phosphorylation by PKA [15-17]. Occasionally, troponin mutations may interfere with the processes involved with thin filament phosphorylation and diminish the subsequent $\mathrm{Ca}^{2+}$ desensitizing effects.

The thin filament undergoes activation due to important associations coordinated by cTnT. The N-terminal region of $\mathrm{cTnT}$ anchors cTn to Tm and this interaction modulates ATPase activation when $\mathrm{Ca}^{2+}$ concentrations achieve threshold levels [18]. Cardiac TnT has two main interactions with $\mathrm{Tm}$. The first is located in the $\mathrm{N}$-terminus of cTnT and is $\mathrm{Ca}^{2+}$ dependent [19]; and the second is located in the Cterminal portion of cTnT which also interacts with $\mathrm{cTnC}$ and $c \operatorname{TnI}[19,20]$. In addition to anchoring cTn to the thin filament, cTnT has a critical role in the activation of actomyosin ATPase activity [21]. When cTnT is combined with cTnI-cTnC, the actomyosin ATPase is activated in a $\mathrm{Ca}^{2+}$-dependent manner. Critical to this process is the region of overlap that exists between TnT1 (residues 1-158) at the head-to-tail junction of two adjacent Tm molecules [22, 23]. These interactions confer cooperativity to muscle activation when $\mathrm{Ca}^{2+}$ reaches threshold levels [24]. Inhibition of contraction is dependent on cTnI; however, in the absence of TnI, both TnT and TnT1 are able to inhibit the actin activated myosin subfragment-1 (S1) ATPase [22, 25].

\section{Restrictive Cardiomyopathy in Humans}

RCM is a rare cardiac disorder that manifests primarily as an abnormality of diastolic filling which is also reported as a reduced ventricular compliance or increased stiffness [26]. This results in a restriction of ventricular filling (reduced diastolic volume) and an increase in end-diastolic pressure while maintaining normal to near normal systolic function [27]. Clinically, RCM presents in patients as dyspnea and pulmonary edema [28]. Generally, both atria become dilated, which is likely due to the restriction of ventricular filling. The heart may not be enlarged and the septal and ventricular wall thickness dimensions are minimally affected [29]. Over time, the systolic function declines as the disease approaches end stages and ultimately results in heart failure [30]. The prognosis of RCM is poor, especially in pediatric cases where heart transplantation is often the only preferred treatment. SCD is prevalent in pediatric RCM with a twoyear mean survival rate for pediatric cases reaching $\sim 50 \%$ after diagnosis [30-33]. SCD is also prevalent in pediatric
RCM cases that display electrocardiographic evidence of ischemia [30].

Several studies confirmed that restrictive cardiomyopathy can be inherited in a familial manner (see Table 1). The first study in 1992 reported the familial inheritance of a disease that resembled RCM and Hypertrophic Cardiomyopathy (HCM) [34]. In 2003, Mogensen and colleagues firmly established that troponin I mutations were etiological causes of RCM [35]. In one case, after evaluating a child and his mother for RCM, examination of the extended family revealed that 12 individuals had died suddenly, with the surviving relatives presenting symptoms of heart disease [35]. Linkage analysis identified a novel missense mutation $(\mathrm{D} 190 \mathrm{H})$ in a conserved region of the cTnI gene (TNNI3) that cosegregated in family members presenting symptoms of cardiac disease. This study also included unrelated patients with idiopathic RCM (IRCM) to assess if a genetic basis could be determined. Genetic analysis of several pediatric IRCM patients identified two different de novo mutations (R192H and K178E) in the TNNI3 gene. Additional patients with adult onset IRCM were also evaluated and the genetic analysis revealed three more mutations in the TNNI3 gene (R145W, A171T, and L144Q) [35]. Notably, these mutations cluster in specific regions of cTnI that participate in important thin filament interactions.

Since 2005, numerous mutations linked to RCM have been identified in the TNNI3 gene. A study utilizing a candidate gene approach identified the deletion of a nucleotide in the TNNI3 gene that causes a frame shift in codon 168 from a 23-year-old patient diagnosed with RCM [36]. This deletion resulted in a premature stop codon at position 176 which leads to the expression of a C-terminal truncated mutant protein. During a six-year follow-up, the patient's heart had undergone progressive and concentric remodeling of the left ventricle. A strong family history was also present as the patient's father had experienced SCD at age 29 and two pregnant relatives experienced intrauterine fetal death in their third trimester. At least two family members under the age of 1 year had suddenly died. The authors established that the frame shift mutation caused a 50\% decrease in the myocardium's total cTnI content while the truncated cTnI was undetectable. These results suggest that the frame shift exerted its deleterious effects through haploinsufficiency. This might be due to cTnI degradation or the failure of the mutant cTnI to associate with the thin filament. In any event, the reduced cTnI content may lead to altered interactions of $\mathrm{cTn}$ with the actin-tropomyosin complex that resulted in severe diastolic dysfunction within this family [36].

In 2008, Kaski et al. identified two other cTnI mutations from two pediatric RCM patients [37]. The first was a de novo substitution (K178E) identified in a 7-year-old girl who had biatrial enlargement, abnormal sinus rhythm and ST-T waves. Histological evaluation of her cardiac tissue revealed cardiomyocyte disarray. The second, identified in a 6-year-old girl, was a novel deletion of two nucleotides that resulted in a frame shift at residue 177 and the occurrence of a premature stop codon at residue 209. Her electrocardiogram also revealed abnormalities in sinus rhythm and 


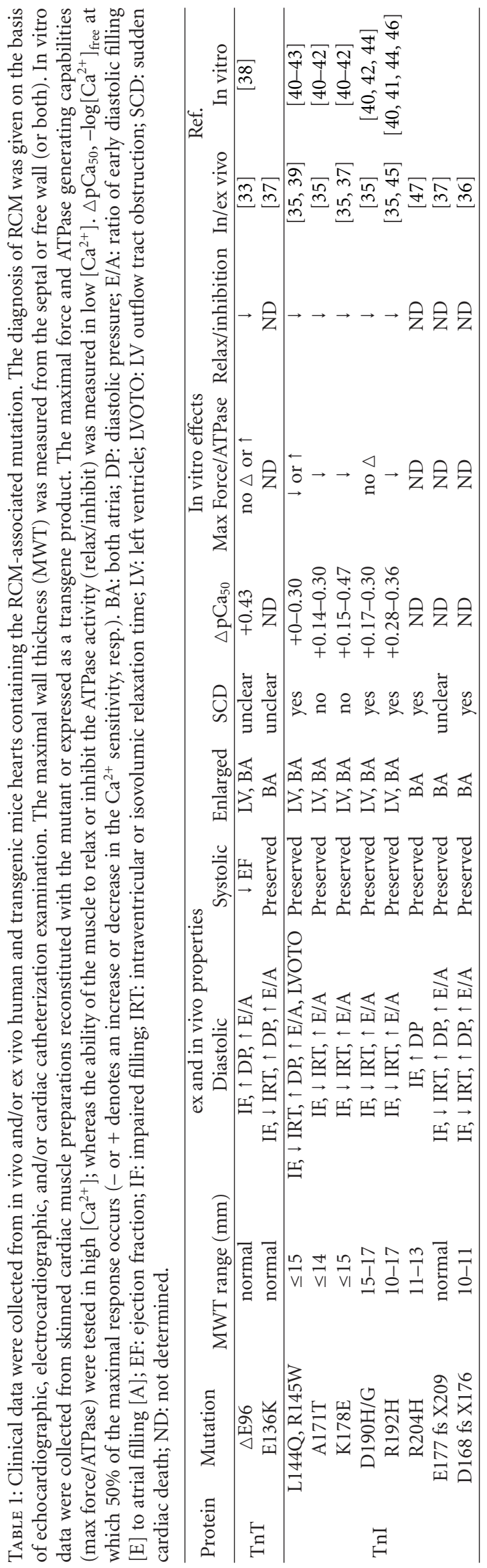


ST-T waves. Morphologically, her heart presented with biatrial enlargement and dysplastic coronaries. Histological evaluation showed cardiomyocyte disarray [37]. The authors suggested that de novo mutations that arise in sarcomeric proteins are generally associated with an earlier onset of the disease and a more deleterious prognosis. These mutations may result from germ line mosaicism [48] or somatic mutations that occur in heart progenitor cells [49].

Another HCM-associated TNNI3 mutation (R204H) was recently identified in a young girl that developed biatrial enlargement during her early teenage years which developed into RCM. Her brother had died suddenly at age 18. Subsequently, she was under clinical observation and developed RCM between the ages of 14-18. The patient had frequent episodes of diastolic heart failure and underwent a heart transplant at age 23. The histopathology performed on her explanted heart showed an absence of hypertrophy, even though myocyte disarray was evident which further supported the RCM diagnosis [47].

Restrictive cardiomyopathy shares some similar clinical features with HCM. For example, both diseases result in the failure of the myocardium to relax fully during diastole. Therefore, the diagnosis of patients with sarcomeric mutations can be confounded when they present with IRCM and HCM. The R192H cTnI mutation identified by Mogensen in 2003 in an RCM patient [35] was also found in another family in 2009 which presented with features of HCM [45]. The proband had a maximal left ventricular wall thickness of $17 \mathrm{~mm}$, a restrictive physiology and a parent and sibling diagnosed with RCM [45]. The R192H mutation is located within the actin binding region of cTnI and is expected to impair cardiac relaxation since it caused a decrease in the left ventricular end diastolic dimension and enlarged the atria in the patient [45]. Generally, RCM and HCM with restrictive physiology caused by mutations in the TNNI3 gene have worse clinical outcomes and disease progression.

Initially, familial RCM occurred primarily in the genes encoding cTnI and cardiac desmin (DES) $[26,35,36,40$, 47]. However, mutations in the $\alpha$-cardiac actin (ACTC), $\beta$ myosin heavy chain ( $\beta$-MHC), and cTnT (TNNT2) genes have recently emerged as etiological causes of RCM $[33,37$, 50]. In 2006, the first mutation in the cTnT gene (TNNT2) linked to RCM was identified in a patient, a deletion of Glu at residue 96 [33]. This twelve-month-old girl was found cyanotic and limp. Upon evaluation, her electrocardiogram (ECG) revealed abnormal sinus rhythm, left axis deviation, and nonspecific changes in the ST-T wave. Echocardiography showed severe atrial dilation with no regurgitation of the mitral and tricuspid valves. In addition, the patient had mild-to-moderate left ventricular systolic dysfunction with a reduced fractional shortening and mild right-ventricular systolic dysfunction. While waiting for transplantation, she experienced recurrent episodes of sinus brachycardia and tachycardia associated with diffuse ischemic ECG changes and pronounced hypertension [33].

Recently, another mutation (E136K) was identified in the TNNT2 gene from a patient diagnosed with RCM [37]. Interestingly, the mutation was also identified in the father and brother, who revealed nothing abnormal upon clinical evaluation. Cardiac tissue from the explanted heart showed that vacuolation was present but was lacking cardiomyocyte disarray normally associated with cTnT-based cardiomyopathies. Since the two relatives were unaffected clinically, the true etiology of the disease remains undetermined. It is possible that another undetected mutation contributes to the phenotype seen in the RCM proband that was absent in the relatives.

As the number of genes associated with RCM has increased, it has become evident that mutations previously determined to cause HCM can also cause RCM or HCM with a restrictive phenotype. A study by Menon (2008) identified a large family displaying cardiac disease inherited in an autosomal dominant pattern. Characterization of the RCM proband showed the existence of massive bi-atrial enlargement and abnormal diastolic function [51]. However, the proband had mild mid-septal hypertrophy without left ventricular outflow obstruction. Interestingly, other relatives presented with clinical features of RCM, HCM, and/or dilated cardiomyopathy (DCM). It was suspected that a single mutation was responsible for the variable disease outcomes; therefore, a targeted linkage analysis was performed for nine sarcomeric genes which determined that the I79N mutation in the TNNT2 gene cosegregated with the disease phenotype. Previously, this mutation was identified in other individuals diagnosed with $\operatorname{HCM}[52,53]$. Studies using transgenic mice containing the I79N TnT mutation have shown that their cardiac function is consistent with a restrictive physiology [54-56].

Other studies have also examined the prevalence and clinical significance of HCM with a restrictive phenotype. Three previously characterized TNNI3 mutations (L144Q, R145W, and D190G) were identified in patients manifesting the restrictive phenotype. HCM is generally associated with mild to moderate disability and normal life expectancies as long as SCD is avoided; however, patients with the "restrictive phenotype" have a very poor prognosis [39]. The restrictive phenotype is characterized as an absence of or minimal hypertrophy with restrictive filling, elevated rates of atrial fibrillation/flutter, diastolic heart failure and stroke. The overall survival rate of these patients is $56 \%$ five years after diagnosis with the main cause of fatality being heart failure [39]. Until recently, it was believed that HCM, DCM and RCM were distinct and separate diseases. However, the R145G and R145W mutations located at the same locus in the cTnI gene can cause HCM or RCM, respectively. This indicates that RCM is included in the spectrum of hereditary cardiac contractile disorders $[35,57]$. To date, the existence of simultaneous multiple mutations, modifier genes or the influence of genetic background has not been fully explored. These aspects will gain increasing interest as knowledge of cardiomyopathies expands in the coming years.

\section{Reconstituted and Structural Assays}

The functional effects of cTnT and cTnI RCM mutations have been explored in different types of reconstituted systems (see Table 1). The in vitro and in situ experimental models involve skinned fibers exchanged with RCM mutant 
proteins, myosin-actin-Tm-cTn reconstituted ATPase assays, and spectroscopic measurements utilizing fluorescence and circular dichroism. Much of this data can be correlated to the clinical observations seen in patients. Furthermore, in addition to linkage analysis, these studies assist in establishing de novo mutations as causative agents of the disease.

4.1. Skinned Fiber Data. Skinned cardiac fiber experiments provide a method of exploring myofilament function with a level of complexity that approaches the intact muscle. These assays are considered one of the most relevant physiological techniques available. Importantly, the sarcomeric proteins at the $\mathrm{M}$ - and Z-band remain in the skinned fiber in contrast to other reconstituted methods. These experiments measure the $\mathrm{Ca}^{2+}$ buffering capacities of the myofilament while measuring the development of tension or maximal force $[58,59]$. Several groups have also reported differences in the level of basal force (at low $\mathrm{Ca}^{2+}$ concentrations) after the incorporation of mutant $\mathrm{cTnT}$ or $\mathrm{cTnI}[38,41]$.

Our group first investigated the functional effects of cTnI mutations linked to RCM [41]. All five RCM mutants that were investigated (L144Q, R145W, A171T, K178E, and $\mathrm{R} 192 \mathrm{H})$ increased the $\mathrm{Ca}^{2+}$ sensitivity of force development in skinned fibers. The maximal tension was diminished for all mutants, except R192H. The L144Q, R145W, and K178E mutants showed a significant increase in the basal force levels compared to WT [41]. Other studies using these mutants also produced a large leftward shift in the $\mathrm{Ca}^{2+}$ sensitivity of force development in exchanged cardiac skinned fibers [40]. The maximal force was reduced in the presence of the L144Q, R145W, A171T, and R192H mutants; however D190G had no effect on the maximal force when compared to the WT [40]. Using adenoviral gene transfer techniques, Davis et al. introduced RCM mutations into cardiomyocytes and studied their functional consequences at the cellular level [42]. In contrast to the previous study [41], skinned rat cardiomyocytes containing the rat cTnI mutations, L145Q and R146W (corresponding to L144Q and R145W), did not show changes in the $\mathrm{Ca}^{2+}$ sensitivity of force development [42]. It is possible that the cTnI mutants located in the inhibitory peptide were poorly incorporated into the thin filament which could mask the effects of the mutations on the $\mathrm{Ca}^{2+}$ sensitivity of contraction. However, the rat cTnI mutations, A172T, K179E and D191G (corresponding to human A171T, K178E, and D190G) increased the $\mathrm{Ca}^{2+}$ sensitivity of force development [42]. Moreover, the transfected cardiomyocytes bearing the mutant cTnIs could be desensitized to $\mathrm{Ca}^{2+}$ upon PKA treatment [42], however, these mutations did not influence the ability of cTnI to respond to adrenergic stimulation. In a separate study, Davis et al. investigated the functional effects of the cTnI R193H mutant incorporated into the thin filament which caused significant $\mathrm{Ca}^{2+}$ sensitization of the myofilaments and slowed the $\mathrm{Ca}^{2+}$ transient decay rate [46].

Previously, we studied the functional consequences of the first cTnT mutation related to RCM where glutamic acid was deleted at position 96 (cTnT- $\triangle \mathrm{E} 96)$ [38]. The skinned fiber measurements showed significant $\mathrm{Ca}^{2+}$ sensitization of the myofilaments and the mutant cTn complex increased the basal force after troponin exchange. The mutation did not affect the maximal force recovery at saturating $\mathrm{Ca}^{2+}$ concentrations. In addition, the same mutation was also studied as the corresponding residue of the fetal isoform of $c \operatorname{TnT}$ (cTnT1). Interestingly, in the fetal environment, i.e., the cTn complex containing the cTnT isoform 1 (cTnT$\Delta \mathrm{E} 106)$ and slow skeletal TnI, the mutation caused a less pronounced effect than when in the adult environment [38]. This suggests that fetal cTn isoforms have a protective role and may explain why the disease occurs early after birth. The mutation significantly reduced the cooperativity (i.e., $\left.n_{\mathrm{Hill}}\right)$ of $\mathrm{Ca}^{2+}$ binding to the regulatory units. This information can be correlated with the previous findings and related to the time of onset and the severity of the disease [60].

4.2. Myosin-Actin-Tm-Tn ATPase Assays. The measurement of myofilament ATPase activity is very important since these experiments indicate the ability of the troponin complex to initiate activation and inhibition of the myosin-actin ATPase activity. The $\mathrm{Ca}^{2+}$ concentration can be adjusted to activate or inhibit the ATPase activity. In other words, this experiment evaluates the influence of Tn in modulating cross-bridge kinetics. Additionally, it is possible to measure the rate of ATP hydrolysis as a function of the free $\mathrm{Ca}^{2+}$ concentration.

In an elegant set of experiments, Gomes et al. showed the dependence of different ratios of WT and mutant cTnI related to RCM on the ability to inhibit the ATPase activity. For example, cTnI R145W in a 50\%:50\% mixture with WT was able to fully inhibit the ATPase; however L144Q in a $50 \%: 50 \%$ ratio with WT had the greatest inability to inhibit ATPase activity and had a dominant regulatory effect over the WT. Regarding the acto-myosin ATPase activation, cTn containing the L144Q mutation partially lost its ability to activate [41]. Kobayashi and Solaro also investigated the functional consequences of the R145W, D190H, and R192H RCM mutations in cTnI. All of the RCM mutants markedly increased the $\mathrm{Ca}^{2+}$ sensitivity of ATPase activity. In addition, the R145W mutant which is located in the inhibitory portion of cTnI showed an increase in the basal ATPase activity at low $\mathrm{Ca}^{2+}$ concentrations [44]. These experimental results suggest that RCM mutations in the inhibitory peptide have a more drastic effect than mutations located in the cTnI C-terminal domain. In the absence of $\mathrm{Ca}^{2+}$, the inhibitory region is very important for maintaining proper thin filament function in the off state.

Thin filaments reconstituted with the first described cTnT mutant $(\triangle \mathrm{E} 96)$ were used to measure actomyosin ATPase activity. In the presence of this mutant, the actomyosin ATPase activity was poorly inhibited at low $\mathrm{Ca}^{2+}$ levels. However, it appears that introducing the mutation into the fetal cTnT isoform in the presence of the fetal slow skeletal TnI diminished these deleterious effects [38].

These functional studies confirm that the RCM phenotype can be directly correlated with clinical data obtained from patients. For example, the increased $\mathrm{Ca}^{2+}$ sensitivity, impaired inhibition of ATPase activity by the Tn complex containing 
RCM mutants, and increased basal force can be directly correlated with diastolic dysfunction.

4.3. Spectroscopy Measurements. Steady-state fluorescence measurements were used to show that the R145W, D190H and $\mathrm{R} 192 \mathrm{H}$ cTnI RCM mutants only increased the apparent $\mathrm{Ca}^{2+}$ binding affinity of $\mathrm{cTnC}$ in the presence of the other thin filament components [44]. Structural studies with NMR using the ${ }^{13} \mathrm{C} /{ }^{15} \mathrm{~N}$-labeled $\mathrm{cTnI}_{129-210}$ peptide containing the K178E RCM mutation revealed local structural changes in the region immediately adjacent to the mutated residue. The cTnI K178E mutant was also evaluated by circular dichroism and no changes were observed in the cTnI K178E structure compared to WT [40]. Together, this data suggests that local yet minor changes in the structure of the isolated mutants may disrupt crucial interactions with other thin filament proteins. In general, the alteration of a few key residues in sites of interactions may be sufficient to alter cTn function.

\section{Transgenic Models}

A specific functional phenotype is emerging for RCM that includes large increases in the $\mathrm{Ca}^{2+}$ sensitivity of tension, impaired ability to inhibit the myosin ATPase activity and increased basal force in skinned fibers. All of these factors when manifested in vivo can contribute to severe diastolic dysfunction and may account for the RCM disease [61]. One of the advantages of using animal models is that the mutant protein is naturally incorporated into the thin filament. Therefore, characterizing the effects of RCM mutations in transgenic or knock-in animals is imperative in determining if cardiac function is affected. Unlike HCM and DCM, the diagnosis of RCM is not based on the morphological appearance of the heart but primarily on the properties of the working heart. Abnormal diastolic filling is the primary defect that leads to increased myocardial stiffness and higher ventricular pressures $[27-29,61]$.

Two transgenic mouse models for cTnI RCM mutations have been reported which investigated the physiological effects of the R192H and R145W mutations. Du et al. designed a transgenic mouse carrying the R193H mutation (which corresponds to human $\mathrm{R} 192 \mathrm{H}$ ) and evaluated its effects on gross cardiac morphology. The transgenic R193H mice demonstrated RCM characteristics, i.e., atrial dilation and decreased left ventricular end-diastolic dimension without significant changes in systolic function. Also, they observed a significant change in the ejection fraction in middle aged (11 months old) mice. Histological evaluation indicated that the cardiac tissue was structurally normal [62]. Functionally, the most significant changes that occurred were impaired relaxation manifested by prolonged IRT (isovolumic relaxation time) and DT (deceleration time). The restrictive physiology was evident by the reduced $\mathrm{E}$ peak velocity and reversed $\mathrm{E} / \mathrm{A}$ on doppler tissue imaging [63]. These observations directly correlated with the clinical phenotype used to establish RCM in a patient.

In another study, Du et al. evaluated the progression of RCM in a cTnI R193H transgenic mouse over a period of 12 months. During this time, cardiac function was assessed to monitor the development of diastolic dysfunction. Initially, the mutation caused abnormal relaxation which gradually manifested as diastolic dysfunction as age progressed. Highresolution echocardiography was used to characterize the mutation's effects on diastolic function by a reversed $\mathrm{E}$ to A ratio, increased DT and prolonged IRT. Some of the cTnI R193H middle-aged mice (1 year) had a significant reduction in cardiac output and a few died from congestive heart failure. Morphologically, the mutant mouse hearts had dilated atria and ventricular restriction similar to those of RCM patients carrying the cTnI R193H mutation. The mutation affected mouse cardiac function in the same manner as seen in the patients by recapitulating the diastolic dysfunction and diastolic heart failure. Altogether, this data suggests that the $\mathrm{C}$-terminus of $\mathrm{cTnI}$ plays an important role in maintaining the diastolic parameters of the heart [63].

The cTnI R145W RCM mouse model was investigated to determine how a different substitution at the same locus which causes HCM (R145G) can lead to two different diseases [57]. In transgenic R145W cardiac myofibrils, an increase in the $\mathrm{Ca}^{2+}$ sensitivity of force and ATPase was observed. Interestingly, the intracellular $\mathrm{Ca}^{2+}$ and force transients measured in intact fibers were delayed which may contribute to the diastolic dysfunction. One possible explanation for the distinct phenotypic differences between RCM and HCM is the presence of an increase in the maximal force as observed only in the R145W mice. The contractility of the ventricular muscle containing the R145W mutant is increased 53\% during systole, thus compensating for the diastolic dysfunction. In contrast, the R145G HCM mice showed a decrease in the maximal force which may explain why the development of cardiac hypertrophy occurs [43].

\section{Isoform Switch, Disease Onset, and Lethality}

During fetal heart development, the expression and presence of different isoforms of cTnI and cTnT varies. These changes in isoform expression may underlie the early onset and aggressive course of pediatric cardiomyopathies. The slow skeletal $\mathrm{TnI}$ isoform is a product of a distinct gene and is expressed and incorporated in the heart during fetal and neonatal development. The effects of mutations in the cardiac isoform of $\mathrm{TnI}$ appear later, as regulated by the activation of cTnI gene expression. It was determined in the rat that $\mathrm{cTnI}$ is expressed by the fourth day after birth and isoform switching continues up to the fourteenth day $[64,65]$. Mutations in the cardiac TnI gene that change cTn function would consequently alter the $\mathrm{Ca}^{2+}$ homeostasis of the cell and manifest their deleterious effects shortly after birth. Cardiac TnT also undergoes an isoform switch. The fetal heart expresses a different isoform by alternative splicing of the TNNT2 gene. The fetal isoform of cTnT (cTnT1) contains both exons 4 and 5 , while the adult isoform (cTnT3) contains only exon 4 [66-68]. When the corresponding RCM mutation ( $\triangle \mathrm{E} 96$ adult isoform $\mathrm{CTnT} 3$ ) was introduced into $\mathrm{CTnT1}$ (i.e., $\triangle \mathrm{E} 106)$ in the presence of slow skeletal TnI, the functional effects were less dramatic [38]. This observation suggests that the combination of fetal cTnT and ssTnI isoforms can rescue the effects of an RCM mutation in 
the cTnT gene. In contrast, throughout cardiac development, cTnC is continually expressed; therefore, it is expected that RCM causing mutations in cTnC may lead to late term or spontaneous abortion. This may explain why mutations in cTnC linked to RCM have not been identified yet.

\section{Structural Consideration and Perspectives}

The two reported cTnT RCM mutations are located in a hot spot region of the TnT N-terminal domain which interfaces with $\mathrm{Tm}$. We speculate that the mutations in $\mathrm{cTnT}$ may disrupt the communication between TnT-Tm and thus affect the actomyosin interaction and cooperative activation of the thin filament. Therefore, future investigations using cTnT RCM mutants may help to dissect the modulatory role and function of the cTnT N-terminal domain.

The RCM mutations found in TnI are located within the inhibitory peptide or within the C-terminal domain. The Cterminal domain of cTnI binds to actin and helps to maintain the thin filament in a blocked state. These cTnI mutations may be destabilizing, i.e., decreasing the interactions between the cTnI C-terminal domain and actin in the absence of $\mathrm{Ca}^{2+}$, consequently relieving cTnI inhibition. The small region termed the inhibitory peptide is necessary for the regulatory role of $\mathrm{cTnI}$ on muscle contraction. It is also possible that mutations located within the cTnI C-terminal domain may alter the structure and function of the inhibitory peptide.

The discovery of new mutations in RCM patients is crucial for dissecting how they alter function and may lead to a better understanding of the disease. The functional and clinical data should be weighed equally in order to better link mutations as causes of cardiomyopathies. In addition, functional studies may help to further define the RCM and HCM phenotypes which share similar features, such as, an increase in the $\mathrm{Ca}^{2+}$ sensitivity of force development. Exploration of RCM causing mutations provides an excellent framework on which new therapeutic strategies can be developed to target the effects of this disease.

\section{Acknowledgments}

This work was supported by NIH Grants HL-67415 and HL-42325 (J. D. Potter) and postdoctoral fellowships from the American Heart Association, AHA 0825368E (for J. R. Pinto) and AHA 09POST2300030 (M. S. Parvatiyar). M. S. Parvatiyar and J. R. Pinto contributed equally to this manuscript; therefore, they are considered cofirst authors.

\section{References}

[1] A. M. Gordon, E. Homsher, and M. Regnier, "Regulation of contraction in striated muscle," Physiological Reviews, vol. 80, no. 2, pp. 853-924, 2000.

[2] A. M. Gordon, M. Regnier, and E. Homsher, "Skeletal and cardiac muscle contractile activation: tropomyosin "rocks and rolls"'” News in Physiological Sciences, vol. 16, no. 2, pp. 49-55, 2001.

[3] B. C. Knollmann, P. Kirchhof, S. G. Sirenko, et al., "Familial hypertrophic cardiomyopathy-linked mutant troponin $\mathrm{T}$ causes stress-induced ventricular tachycardia and
$\mathrm{Ca}^{2+}$-dependent action potential remodeling," Circulation Research, vol. 92, no. 4, pp. 428-436, 2003.

[4] M. X. Li, L. Spyracopoulos, and B. D. Sykes, "Binding of cardiac troponin-I 147-163 induces a structural opening in human cardiac troponin-C," Biochemistry, vol. 38, no. 26, pp. 8289-8298, 1999.

[5] S. Takeda, A. Yamashita, K. Maeda, and Y. Maéda, "Structure of the core domain of human cardiac troponin in the $\mathrm{Ca}^{2+}$ saturated form," Nature, vol. 424, no. 6944, pp. 35-41, 2003.

[6] C. S. Farah, C. A. Miyamoto, C. H. I. Ramos, et al., "Structural and regulatory functions of the $\mathrm{NH}_{2}$ - and $\mathrm{COOH}$-terminal regions of skeletal muscle troponin I," Journal of Biological Chemistry, vol. 269, no. 7, pp. 5230-5240, 1994.

[7] K. Murakami, F. Yumoto, S.-Y. Ohki, T. Yasunaga, M. Tanokura, and T. Wakabayashi, "Structural basis for $\mathrm{Ca}^{2+}$ regulated muscle relaxation at interaction sites of troponin with actin and tropomyosin," Journal of Molecular Biology, vol. 352, no. 1, pp. 178-201, 2005.

[8] C. A. Morris, L. S. Tobacman, and E. Homsher, "Modulation of contractile activation in skeletal muscle by a calciuminsensitive troponin C mutant," Journal of Biological Chemistry, vol. 276, no. 23, pp. 20245-20251, 2001.

[9] D. F. A. McKillop and M. A. Geeves, "Regulation of the interaction between actin and myosin subfragment 1 : evidence for three states of the thin filament," Biophysical Journal, vol. 65, no. 2, pp. 693-701, 1993.

[10] A. J. G. Moir, R. J. Solaro, and S. V. Perry, "The site of phosphorylation of troponin I in the perfused rabbit heart. The effect of adrenaline," Biochemical Journal, vol. 185, no. 2, pp. 505-513, 1980.

[11] T. A. Noland Jr., X. Guo, R. L. Raynor, et al., "Cardiac troponin I mutants. Phosphorylation by protein kinases $\mathrm{C}$ and $\mathrm{A}$ and regulation of $\mathrm{Ca}^{2+}$-stimulated MgATPase of reconstituted actomyosin S-1," Journal of Biological Chemistry, vol. 270, no. 43, pp. 25445-25454, 1995.

[12] J. C. Kentish, D. T. McCloskey, J. Layland, et al., "Phosphorylation of troponin I by protein kinase A accelerates relaxation and crossbridge cycle kinetics in mouse ventricular muscle," Circulation Research, vol. 88, no. 10, pp. 1059-1065, 2001.

[13] K. P. Ray and P. J. England, "Phosphorylation of the inhibitory subunit of troponin and its effects on the calcium dependence of cardiac myofibril adenosine triphosphatase," FEBS Letters, vol. 70, no. 1, pp. 11-16, 1976.

[14] R. J. Solaro, A. J. G. Moir, and S. V. Perry, "Phosphorylation of troponin I and the inotropic effect of adrenaline in the perfused rabbit heart," Nature, vol. 262, no. 5569, pp. 615-617, 1976.

[15] R. Zhang, J. Zhao, A. Mandveno, and J. D. Potter, "Cardiac troponin I phosphorylation increases the rate of cardiac muscle relaxation," Circulation Research, vol. 76, no. 6, pp. 1028-1035, 1995.

[16] D. E. Michele, M. L. Szatkowski, F. P. Albayya, and J. M. Metzger, "Parvalbumin gene delivery improves diastolic function in the aged myocardium in vivo," Molecular Therapy, vol. 10, no. 2, pp. 399-403, 2004.

[17] R. Zhang, J. Zhao, and J. D. Potter, "Phosphorylation of both serine residues in cardiac troponin $\mathrm{I}$ is required to decrease the $\mathrm{Ca}^{2+}$ affinity of cardiac troponin C," Journal of Biological Chemistry, vol. 270, no. 51, pp. 30773-30780, 1995.

[18] B. Malnic, C. S. Farah, and F. C. Reinach, "Regulatory properties of the $\mathrm{NH}_{2}$ - and $\mathrm{COOH}$-terminal domains of troponin T: ATPase activation and binding to troponin I and troponin C," Journal of Biological Chemistry, vol. 273, no. 17, pp. 10594-10601, 1998. 
[19] J. R. Pearlstone and L. B. Smillie, "Effects of troponin-I plus$\mathrm{C}$ on the binding of troponin-T and its fragments to $\alpha$ tropomyosin. $\mathrm{Ca}^{2+}$ sensitivity and cooperativity," Journal of Biological Chemistry, vol. 258, no. 4, pp. 2534-2542, 1983.

[20] S. P. White, C. Cohen, and G. N. Phillips Jr., "Structure of cocrystals of tropomyosin and troponin," Nature, vol. 325, no. 6107, pp. 826-828, 1987.

[21] A. S. Zot and J. D. Potter, "Structural aspects of troponintropomyosin regulation of skeletal muscle contraction," Annual Review of Biophysics and Biophysical Chemistry, vol. 16, pp. 535-559, 1987.

[22] R. Maytum, M. A. Geeves, and S. S. Lehrer, "A modulatory role for the troponin $\mathrm{T}$ tail domain in thin filament regulation," Journal of Biological Chemistry, vol. 277, no. 33, pp. 2977429780, 2002.

[23] L. S. Tobacman, "Structure-function studies of the aminoterminal region of bovine cardiac troponin T," Journal of Biological Chemistry, vol. 263, no. 6, pp. 2668-2672, 1988.

[24] A. Hinkle, A. Goranson, C. A. Butters, and L. S. Tobacman, "Roles for the troponin tail domain in thin filament assembly and regulation: a deletional study of cardiac troponin T," Journal of Biological Chemistry, vol. 274, no. 11, pp. 71577164, 1999.

[25] L. S. Tobacman, M. Nihli, C. Butters, et al., "The troponin tail domain promotes a conformational state of the thin filament that suppresses myosin activity," Journal of Biological Chemistry, vol. 277, no. 31, pp. 27636-27642, 2002.

[26] X.-P. Huang and J.-F. Du, "Troponin I, cardiac diastolic dysfunction and restrictive cardiomyopathy," Acta Pharmacologica Sinica, vol. 25, no. 12, pp. 1569-1575, 2004.

[27] S. E. Hughes and W. J. McKenna, "New insights into the pathology of inherited cardiomyopathy," Heart, vol. 91, no. 2, pp. 257-264, 2005.

[28] A. Angelini, V. Calzolari, G. Thiene, et al., "Morphologic spectrum of primary restrictive cardiomyopathy," American Journal of Cardiology, vol. 80, no. 8, pp. 1046-1050, 1997.

[29] S. S. Kushwaha, J. T. Fallon, and V. Fuster, "Restrictive cardiomyopathy," The New England Journal of Medicine, vol. 336, no. 4, pp. 267-276, 1997.

[30] S. M. Rivenes, D. L. Kearney, E. O. Smith, J. A. Towbin, and S. W. Denfield, "Sudden death and cardiovascular collapse in children with restrictive cardiomyopathy," Circulation, vol. 102, no. 8, pp. 876-882, 2000.

[31] S.-C. Chen, I. C. Balfour, and S. Jureidini, "Clinical spectrum of restrictive cardiomyopathy in children," Journal of Heart and Lung Transplantation, vol. 20, no. 1, pp. 90-92, 2001.

[32] M. T. Kimberling, D. T. Balzer, R. Hirsch, E. Mendeloff, C. B. Huddleston, and C. E. Canter, "Cardiac transplantation for pediatric restrictive cardiomyopathy: presentation, evaluation, and short-term outcome," Journal of Heart and Lung Transplantation, vol. 21, no. 4, pp. 455-459, 2002.

[33] S. B. Peddy, L. A. Vricella, J. E. Crosson, et al., "Infantile restrictive cardiomyopathy resulting from a mutation in the cardiac troponin T gene," Pediatrics, vol. 117, no. 5, pp. 1830$1833,2006$.

[34] S. Feld and A. Caspi, "Familial cardiomyopathy with variable hypertrophic and restrictive features and common HLA haplotype," Israel Journal of Medical Sciences, vol. 28, no. 5, pp. 277-280, 1992.

[35] J. Mogensen, T. Kubo, M. Duque, et al., "Idiopathic restrictive cardiomyopathy is part of the clinical expression of cardiac troponin I mutations," Journal of Clinical Investigation, vol. 111, no. 2, pp. 209-216, 2003.
[36] A. Kostareva, A. Gudkova, G. Sjoberg, et al., "Deletion in TNNI3 gene is associated with restrictive cardiomyopathy," International Journal of Cardiology, vol. 131, no. 3, pp. 410412, 2009.

[37] J. P. Kaski, P. Syrris, M. Burch, et al., "Idiopathic restrictive cardiomyopathy in children is caused by mutations in cardiac sarcomere protein genes," Heart, vol. 94, no. 11, pp. 14781484, 2008.

[38] J. R. Pinto, M. S. Parvatiyar, M. A. Jones, J. Liang, and J. D. Potter, "A troponin T mutation that causes infantile restrictive cardiomyopathy increases $\mathrm{Ca}^{2+}$ sensitivity of force development and impairs the inhibitory properties of troponin," Journal of Biological Chemistry, vol. 283, no. 4, pp. 2156-2166, 2008.

[39] T. Kubo, J. R. Gimeno, A. Bahl, et al., "Prevalence, clinical significance, and genetic basis of hypertrophic cardiomyopathy with restrictive phenotype," Journal of the American College of Cardiology, vol. 49, no. 25, pp. 2419-2426, 2007.

[40] F. Yumoto, Q.-W. Lu, S. Morimoto, et al., "Drastic $\mathrm{Ca}^{2+}$ sensitization of myofilament associated with a small structural change in troponin I in inherited restrictive cardiomyopathy," Biochemical and Biophysical Research Communications, vol. 338, no. 3, pp. 1519-1526, 2005.

[41] A. V. Gomes, J. Liang, and J. D. Potter, "Mutations in human cardiac troponin I that are associated with restrictive cardiomyopathy affect basal ATPase activity and the calcium sensitivity of force development," Journal of Biological Chemistry, vol. 280, no. 35, pp. 30909-30915, 2005.

[42] J. Davis, H. Wen, T. Edwards, and J. M. Metzger, "Allele and species dependent contractile defects by restrictive and hypertrophic cardiomyopathy-linked troponin I mutants," Journal of Molecular and Cellular Cardiology, vol. 44, no. 5, pp. 891-904, 2008.

[43] Y. Wen, Y. Xu, Y. Wang, J. R. Pinto, J. D. Potter, and W. G. L. Kerrick, "Functional effects of a restrictive-cardiomyopathylinked cardiac troponin I mutation (R145W) in transgenic mice," Journal of Molecular Biology, vol. 392, no. 5, pp. 11581167, 2009.

[44] T. Kobayashi and R. J. Solaro, "Increased $\mathrm{Ca}^{2+}$ affinity of cardiac thin filaments reconstituted with cardiomyopathyrelated mutant cardiac troponin I," Journal of Biological Chemistry, vol. 281, no. 19, pp. 13471-13477, 2006.

[45] T. S. Rai, S. Ahmad, T. S. Ahluwalia, et al., "Genetic and clinical profile of Indian patients of idiopathic restrictive cardiomyopathy with and without hypertrophy," Molecular and Cellular Biochemistry, vol. 331, no. 1-2, pp. 187-192, 2009.

[46] J. Davis, H. Wen, T. Edwards, and J. M. Metzger, "Thin filament disinhibition by restrictive cardiomyopathy mutant $\mathrm{R} 193 \mathrm{H}$ troponin I induces $\mathrm{Ca}^{2+}$-independent mechanical tone and acute myocyte remodeling," Circulation Research, vol. 100, no. 10, pp. 1494-1502, 2007.

[47] F. I. Gambarin, M. Tagliani, and E. Arbustini, "Pure restrictive cardiomyopathy associated with cardiac troponin I gene mutation: mismatch between the lack of hypertrophy and the presence of disarray," Heart, vol. 94, no. 10, p. 1257, 2008.

[48] J.-F. Forissier, P. Richard, S. Briault, et al., "First description of germline mosaicism in familial hypertrophic cardiomyopathy," Journal of Medical Genetics, vol. 37, no. 2, pp. 132-134, 2000.

[49] H. Watkins, L. Thierfelder, D.-S. Hwang, W. McKenna, J. G. Seidman, and C. E. Seidman, "Sporadic hypertrophic cardiomyopathy due to de novo myosin mutations," Journal of Clinical Investigation, vol. 90, no. 5, pp. 1666-1671, 1992. 
[50] S. Karam, M.-J. Raboisson, C. Ducreux, et al., "A de novo mutation of the beta cardiac myosin heavy chain gene in an infantile restrictive cardiomyopathy," Congenital Heart Disease, vol. 3, no. 2, pp. 138-143, 2008.

[51] S. C. Menon, V. V. Michels, P. A. Pellikka, et al., "Cardiac troponin $\mathrm{T}$ mutation in familial cardiomyopathy with variable remodeling and restrictive physiology," Clinical Genetics, vol. 74, no. 5, pp. 445-454, 2008.

[52] H. Watkins, W. J. McKenna, L. Thierfelder, et al., "Mutations in the genes for cardiac troponin $\mathrm{T}$ and $\alpha$-tropomyosin in hypertrophic cardiomyopathy," The New England Journal of Medicine, vol. 332, no. 16, pp. 1058-1064, 1995.

[53] A. M. Varnava, P. M. Elliott, C. Baboonian, F. Davison, M. J. Davies, and W. J. McKenna, "Hypertrophic cardiomyopathy: histopathological features of sudden death in cardiac troponin T disease," Circulation, vol. 104, no. 12, pp. 1380-1384, 2001.

[54] E. M. Rust, F. P. Albayya, and J. M. Metzger, "Identification of a contractile deficit in adult cardiac myocytes expressing hypertrophic cardiomyopathy-associated mutant troponin $\mathrm{T}$ proteins," Journal of Clinical Investigation, vol. 103, no. 10, pp. 1459-1467, 1999.

[55] T. Miller, D. Szczesna, P. R. Housmans, et al., "Abnormal contractile function in transgenic mice expressing a familial hypertrophic cardiomyopathy-linked troponin T (I79N) mutation," Journal of Biological Chemistry, vol. 276, no. 6, pp. 3743-3755, 2001.

[56] D. Westermann, B. C. Knollmann, P. Steendijk, et al., "Diltiazem treatment prevents diastolic heart failure in mice with familial hypertrophic cardiomyopathy," European Journal of Heart Failure, vol. 8, no. 2, pp. 115-121, 2006.

[57] Y. Wen, J. R. Pinto, A. V. Gomes, et al., "Functional consequences of the human cardiac troponin I hypertrophic cardiomyopathy mutation R145G in transgenic mice," Journal of Biological Chemistry, vol. 283, no. 29, pp. 20484-20494, 2008.

[58] D. S. Wood, M. M. Sorenson, A. B. Eastwood, W. E. Charash, and J. P. Reuben, "Duchenne dystrophy: abnormal generation of tension and $\mathrm{Ca}^{++}$regulation in single skinned fibers," Neurology, vol. 28, no. 5, pp. 447-457, 1978.

[59] D. S. Wood, J. Zollman, J. P. Reuben, and P. W. Brandt, "Human skeletal muscle: properties of the 'chemically skinned' fiber," Science, vol. 187, no. 4181, pp. 1075-1076, 1975.

[60] J. Mogensen and E. Arbustini, "Restrictive cardiomyopathy," Current Opinion in Cardiology, vol. 24, no. 3, pp. 214-220, 2009.

[61] N. M. Ammash, J. B. Seward, K. R. Bailey, W. D. Edwards, and A. J. Tajik, "Clinical profile and outcome of idiopathic restrictive cardiomyopathy," Circulation, vol. 101, no. 21, pp. 2490-2496, 2000.

[62] J. Du, C. Zhang, J. Liu, C. Sidky, and X. P. Huang, "A point mutation $(\mathrm{R} 192 \mathrm{H})$ in the C-terminus of human cardiac troponin I causes diastolic dysfunction in transgenic mice," Archives of Biochemistry and Biophysics, vol. 456, no. 2, pp. 143-150, 2006.

[63] J. Du, J. Liu, H.-Z. Feng, et al., "Impaired relaxation is the main manifestation in transgenic mice expressing a restrictive cardiomyopathy mutation, $\mathrm{R} 193 \mathrm{H}$, in cardiac TnI," American Journal of Physiology, vol. 294, no. 6, pp. H2604-H2613, 2008.

[64] A. M. Murphy, L. Jones II, H. F. Sims, and A. W. Strauss, "Molecular cloning of rat cardiac troponin I and analysis of troponin I isoform expression in developing rat heart," Biochemistry, vol. 30, no. 3, pp. 707-712, 1997.
[65] J. M. Metzger, D. E. Michele, E. M. Rust, A. R. Borton, and M. V. Westfall, "Sarcomere thin filament regulatory isoforms: evidence of a dominant effect of slow skeletal troponin I on cardiac contraction," Journal of Biological Chemistry, vol. 278, no. 15, pp. 13118-13123, 2003.

[66] S. V. Perry, "Troponin T: genetics, properties and function," Journal of Muscle Research and Cell Motility, vol. 19, no. 6, pp. 575-602, 1998.

[67] L. S. Tobacman and R. Lee, "Isolation and functional comparison of bovine cardiac troponin T isoforms," Journal of Biological Chemistry, vol. 262, no. 9, pp. 4059-4064, 1987.

[68] A. V. Gomes, G. Guzman, J. Zhao, and J. D. Potter, "Cardiac troponin $\mathrm{T}$ isoforms affect the $\mathrm{Ca}^{2+}$ sensitivity and inhibition of force development: insights into the role of troponin $\mathrm{T}$ isoforms in the heart," Journal of Biological Chemistry, vol. 277, no. 38, pp. 35341-35349, 2002. 

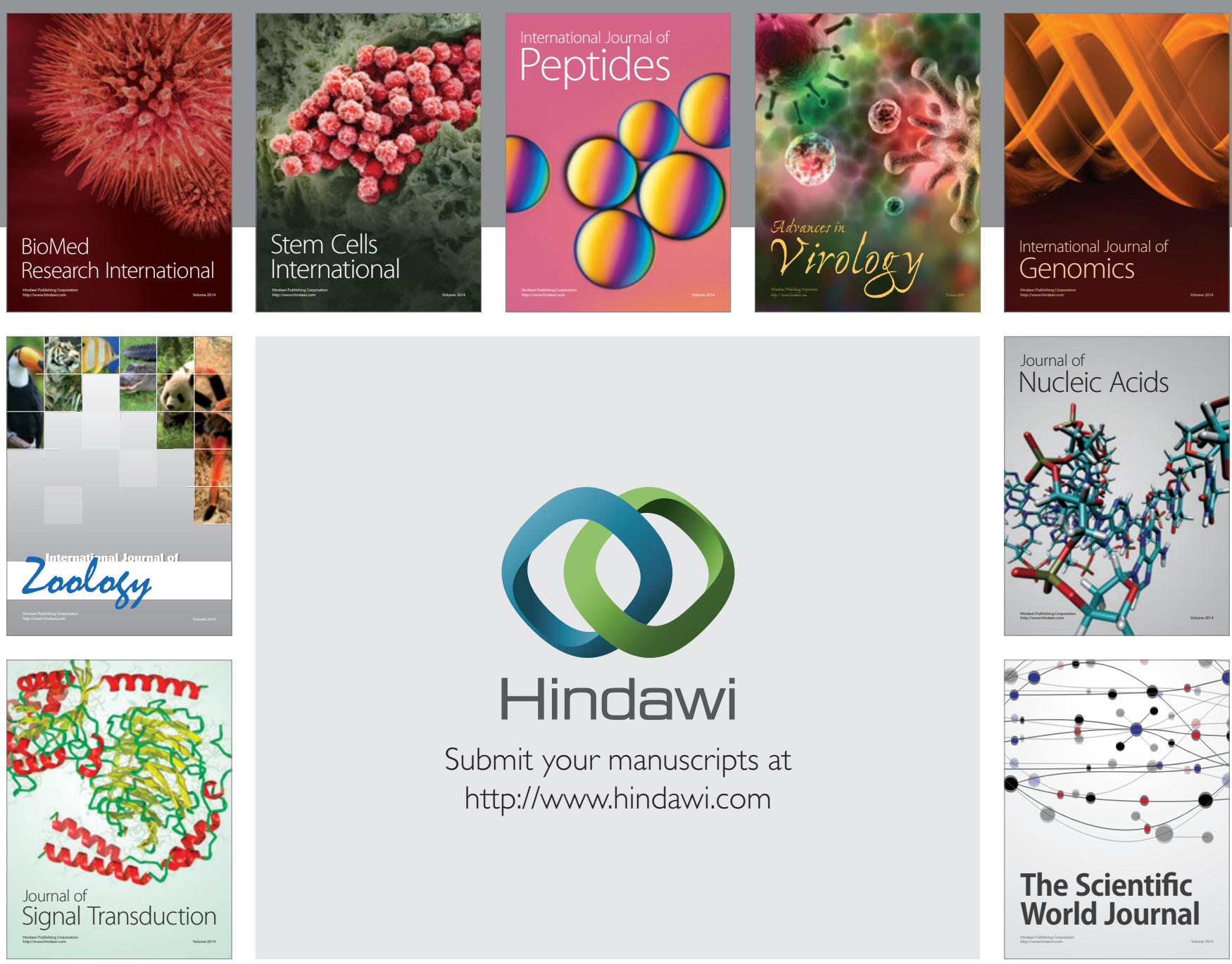

Submit your manuscripts at

http://www.hindawi.com
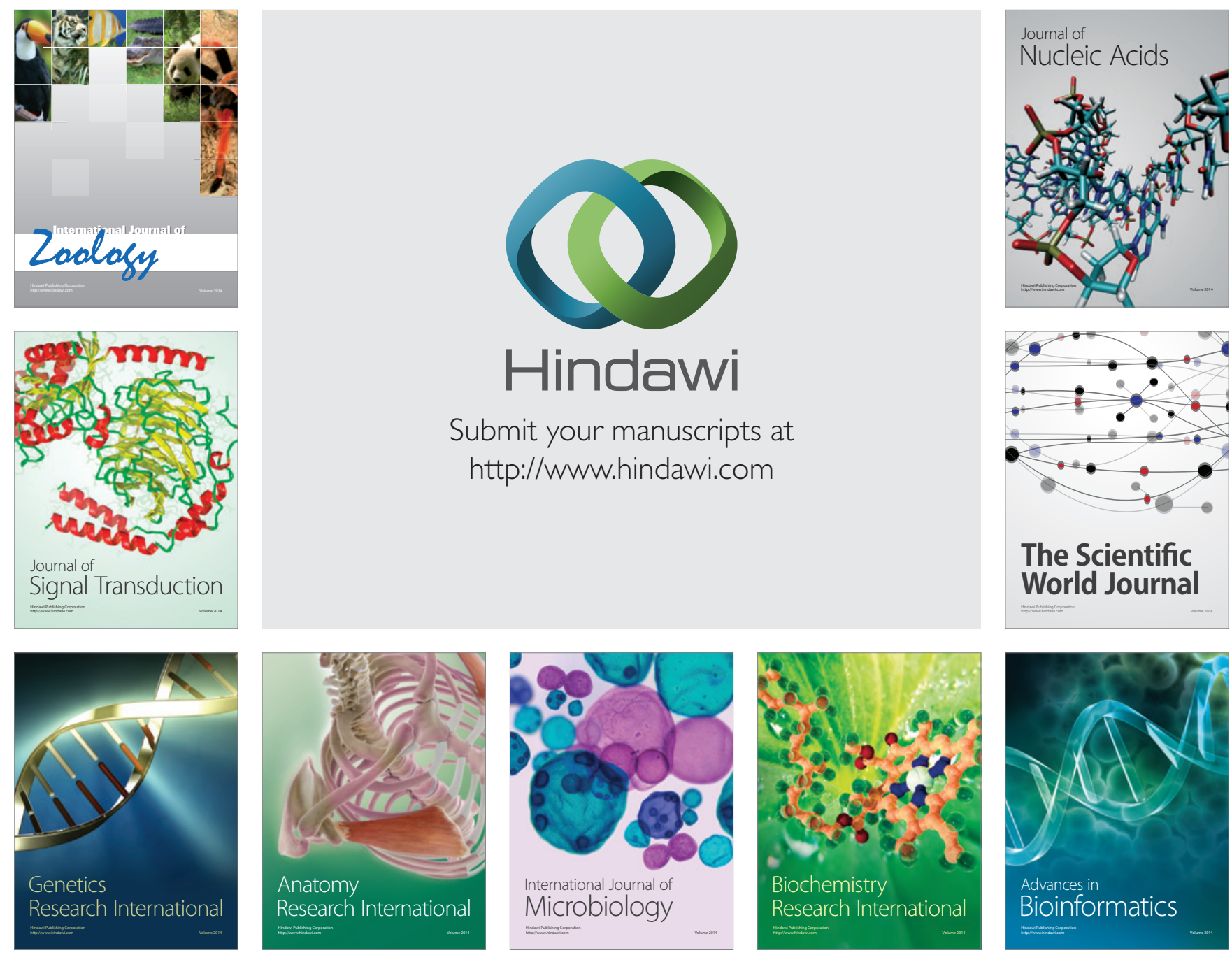

The Scientific World Journal
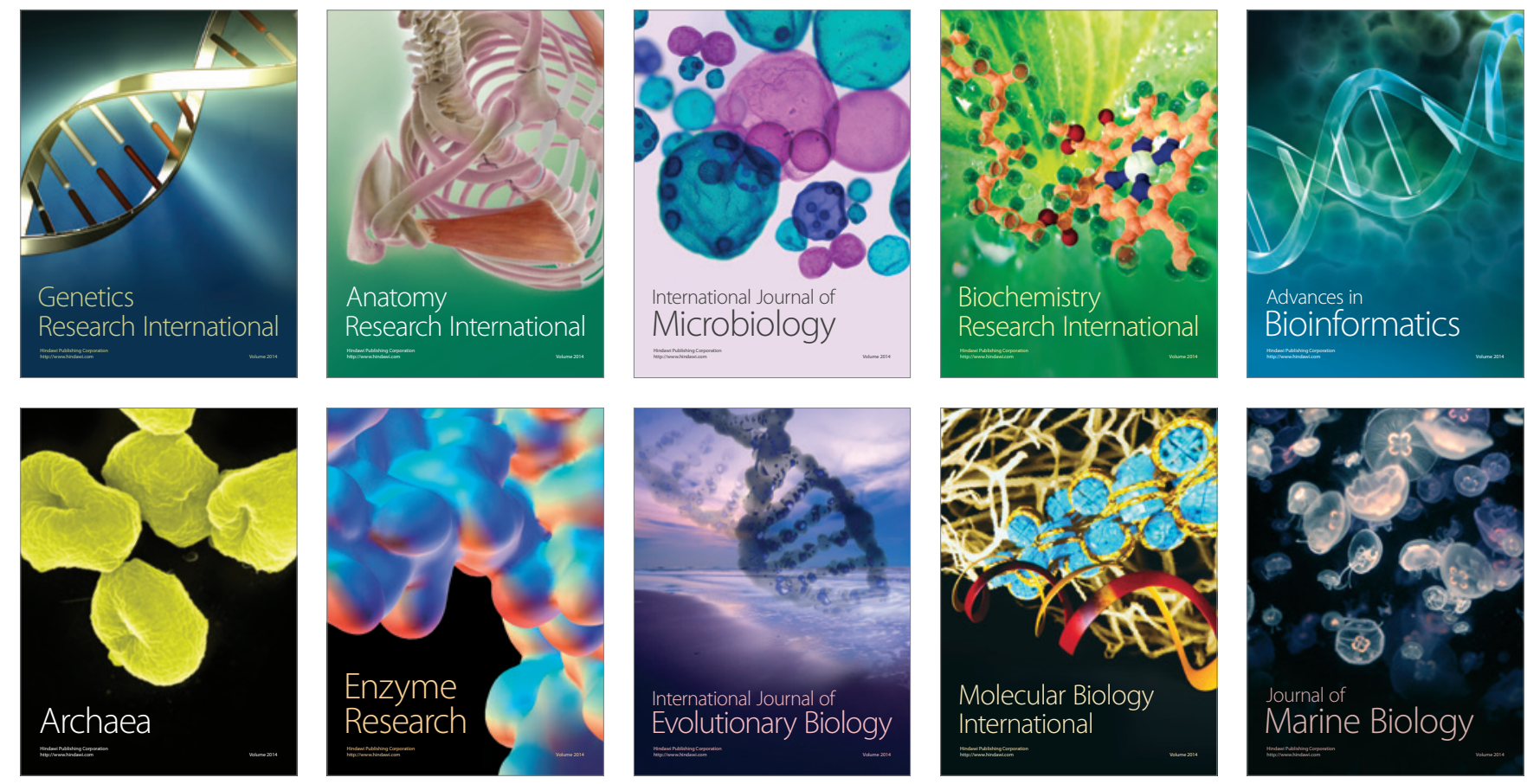